\title{
The Pathway Tracing Method for Solving Facility Location Problem Between Urban Inner and Outer Ring Expressway
}

\author{
YANG Tao ${ }^{1, a}$ \\ ${ }^{1}$ Dept. of Management Science \& Engineering, Shanghai Second Polytechnic University, China \\ ayangtao@sspu.edu.cn
}

\begin{abstract}
Keywords: Facility location, Pathway tracing method, Homotopy pathway.
Abstract. Facility location problems arise for planning and stationing serve centers. The solution of facility location problem can be modeled by a fixed point model. The hard point is that the collection of alternative sites is non-convex set while solving facility location problem between urban inner and outer ring expressway. Commonly used method for solving fixed point model requires the definition set of variables is a convex set. The contribution of this paper is to apply the theorem of existence of the solution of fixed point problem to discuss the facility location problem in this kind of non-convex set and find the numerical result by the pathway tracing method. Numerical example shows that the numerical method by tracing the homotopy pathway with predictor-corrector is an effective method to solving the facility location problem and a global optimal solution can be obtained.
\end{abstract}

\section{Introduction}

Facility location problems arise for siting serve centers including airports, logistics hub, hospitals, distribution centers, post offices, and fire stations. A real facility location problem can be transferred into a mathematical problem to find an optimal point within a collection of alternative sites. The objective of the facility location problem is to minimize travel cost to visit [1] or maximize accessibility of the facility location [2]. The alternative sites can be expressed by the constraint set with equations and inequalities.

With the difficulties of the objective function, there is not a formula to be found for the closed-form solution of the optimizing problem. But the recursive equations generating a succession are adopted, so an equivalent fixed point problem can be referred which solution could be convergence by means of iterations $[3,4]$.

Commonly, the algorithm solving this fixed point model needs that the definition set of variables must be a convex set. The hard point is that the collection of alternative sites is non-convex set while solving facility location problem between urban inner and outer ring expressway. So the assumption is not valid in our problem because the constraint sets is non-convex. The algorithm of the pathway tracing methods for fixed point problem within the non-convex set is discussed along with the theorem of existence of the solution of this kind of fixed point problem [5,6]. Numerical example shows that the numerical method tracing the homotopy pathway with predictor-corrector is an effective method to solving the facility location problem [7] .

\section{Estimation of Transportation Cost}

Suppose $n$ customers are sited in a two dimensional plane, the coordinates of customer $i$ is $c_{i}\left(x_{1 i}, x_{2 i}\right)$. Let the customer $i$ 's demand be $w_{i}$, the freight rate per unit volume and unit distance from the customer $i$ to the facility be $r_{i}$. Suppose a serving facility locate at a point $q\left(x_{1}, x_{2}\right)$. For the objective is to minimize total travel cost to visit the facility location, the transportation distance from $c_{i}$ to $q$ should be estimated firstly.

The Euclidean distance for two points $c_{i}\left(x_{1 i}, x_{2 i}\right)$ and $c_{j}\left(x_{1 j}, x_{2 j}\right)$ in a two dimensional plane is given by the expression $\sqrt{\left(x_{1 i}-x_{1 j}\right)^{2}+\left(x_{2 i}-x_{2 j}\right)^{2}}$. Suppose the urban traffic system is square grid networks, the 
transportation distance from $c_{i}$ to $q$, i.e., the length of the journey from $c_{i}$ to $q$, is determined by the coordinates and their the line direction between the two points.

When the line between the two points makes angle of $j \pi / 2(j=0,1,2,3)$ with axis, the transportation distance is its Euclidean distance. The transportation distance is the Euclidean distance multiplied by $\sqrt{2}$. The mean transportation distance $\bar{d}_{i j}$ for any two points $c_{i}\left(x_{1 i}, x_{2 i}\right)$ and $c_{j}\left(x_{1 j}, x_{2 j}\right)$ is as Eq. 1 .

$$
\bar{d}_{i j}=\frac{4}{\pi} \sqrt{\left(x_{1 i}-x_{1 j}\right)^{2}+\left(x_{2 i}-x_{2 j}\right)^{2}} \approx 1.27 \sqrt{\left(x_{1 i}-x_{1 j}\right)^{2}+\left(x_{2 i}-x_{2 j}\right)^{2}}
$$

That is to say, the transportation distance could be approximated by the Euclidean distance multiplied by a constant which is called the transportation distance factor as follows. Then the traffic cost is the transportation distance $\bar{d}_{i j}$ multiplied by the demand $w_{i}$ and the freight rate $r_{i}$. And then the total travel cost for the facility location point $j$ is to sum up the traffic cost for all the demand point $i$.

\section{Fixed Point Model of Facility Location Problem}

The mathematical optimizing method is commonly applied to model the facility location problems. Suppose the $\Omega$ is the set of alternative sites of the facility, which can be expressed by the constraint set with equations and inequalities. The aim is to locate a serving facility in the set $\Omega$, such that the total travel cost for customers to travel to the facility is minimized. The simple formula to measure the total transportation cost $t$ is as Eq. 2. $\rho$ is identical transportation distance factor of customers,

$$
t\left(x_{1}, x_{2}\right)=\sum_{i=1}^{n} r_{i} w_{i} \boldsymbol{\rho} \sqrt{\left(x_{1}-x_{1 i}\right)^{2}+\left(x_{2}-x_{2 i}\right)^{2}} .
$$

Let $\left(x_{1}^{*}, x_{2}^{*}\right)$ be the optimal facility location points, they must be the solutions of the optimization problem

$$
\left(x_{1}^{*}, x_{2}^{*}\right)=\arg \min t\left(x_{1}, x_{2}\right), \quad\left(x_{1}, x_{2}\right) \in \Omega
$$

With the difficulties of the objective function, there is not a formula to be found for the closed-form solution of the optimizing problem as Eq. 3. However, according to the equations of first-order necessarily $K-K-T$ condition, the original optimization problem as Eq. 3 can be transferred into an equivalent fixed point problem as Eq. 4.

$$
\left(x_{1}^{*}, x_{2}^{*}\right)=\left(\frac{\sum_{i=1}^{n} \frac{r_{i} w_{i} x_{1 i}}{\sqrt{\left(x_{1}^{*}-x_{1 i}\right)^{2}+\left(x_{2}^{*}-x_{2 i}\right)^{2}}}}{\sum_{i=1}^{n} \frac{r_{i} w_{i}}{\sqrt{\left(x_{1}^{*}-x_{1 i}\right)^{2}+\left(x_{2}^{*}-x_{2 i}\right)^{2}}}}, \frac{\sum_{i=1}^{n} \frac{r_{i} w_{i} x_{2 i}}{\sqrt{\left(x_{1}^{*}-x_{1 i}\right)^{2}+\left(x_{2}^{*}-x_{2 i}\right)^{2}}}}{\sqrt{\left(x_{1}^{*}-x_{1 i}\right)^{2}+\left(x_{2}^{*}-x_{2 i}\right)^{2}}}\right), \quad\left(x_{1}^{*}, x_{2}^{*}\right) \in \Omega .
$$

The recursive equations generating a succession are adopted and asymptotically convergent solution can be obtained by means of iterations [5].

The properties of the nonlinear equations system depend on the characteristics of the functions involved and the set $[6,7]$.

\section{The Pathway Tracing Method for Solution of Fixed Point Model}

Existence on the Solution of Fixed Point Model. Commonly, the algorithm solving this fixed point model needs that the definition set of variables must be a convex set as in Brower's theorem. Note that 
the hard point is that the collection of alternative sites is non-convex set while solving facility location problem between urban inner and outer ring expressway.

In general, let $\boldsymbol{\varphi}(\boldsymbol{x})$ be an $n$-vectorial functions of a vector $\boldsymbol{x}$ defined in a set $\Omega \subseteq R^{n}$, with values in the set $T=\varphi(\Omega)=\{\varphi(x): x \in \Omega\} \subseteq R^{n}$; the point $x^{*} \in \Omega$ is called a fixed-point if the function has a value equal to the argument: $x^{*}=\varphi\left(x^{*}\right): x^{*} \in \Omega$.

Weaker conditions that definition set can be extended to the non-convex bounded set $\Omega$ for the fixed point problem are given by a theorem based upon a combined homotopy interior-point method presented by $\mathrm{Yu}$ and Lin [6] which describes an asymptotically convergent resolutive algorithm. Set

$$
\Omega^{0}=\left\{\boldsymbol{x} \in R^{n}: \boldsymbol{g}_{i}(\boldsymbol{x})<0, i=1, \mathrm{~K} m\right\} \subset R^{n} .
$$

and $\Omega$ is a closure of $\Omega^{0}, \boldsymbol{g}=\left(g_{1}, \ldots, g_{m}\right)^{T}, \boldsymbol{Y}=\operatorname{diag}(\boldsymbol{y}), \nabla g_{i}(\boldsymbol{x})^{T}=\frac{\partial g_{i}(\boldsymbol{x})}{\partial \boldsymbol{x}}, B(\boldsymbol{x})=\left\{i \in\{1, \mathrm{~K}, m\}: g_{i}(\boldsymbol{x})=0\right\}$ is the active index set at $\boldsymbol{x}$. Then the main results of paper [6,7] are as follows

Theorem 1. Suppose all $g_{i}(\boldsymbol{x})$ are three times continuously differentiable functions, and

(i) $\Omega^{0}$ is nonempty and bounded;

(ii) $\forall \boldsymbol{x} \in \partial \Omega,\left\{\nabla g_{i}(\boldsymbol{x}): i \in B(\boldsymbol{x})\right\}$ is of full column rank;

(iii) The normal cone of $\Omega$ at any only meets $\Omega$ at $\boldsymbol{x}$, i.e., for any $\boldsymbol{x} \in \partial \Omega$

$$
\left\{\boldsymbol{x}+\sum_{i \in B(x)} y_{i} g_{i}(\boldsymbol{x}): y_{i} \geq 0, i \in B(\boldsymbol{x}), \sum_{i \in B(\boldsymbol{x})} y_{i}>0\right\} \cap \Omega=\{\boldsymbol{x}\}
$$

Then the fixed points of $\varphi(x)$ in $\Omega$ correspond precisely to the solutions of the system

$$
\begin{aligned}
& x-\varphi(x)+\nabla g(x) y=0, \\
& Y g(x)=0, g(x) \leq 0, y \geq 0 .
\end{aligned}
$$

And for almost all starting points $(x, y) \in \Omega^{0} \times R_{++}^{m}$, there is a regular solution curve of homotopy

$$
\begin{aligned}
& (1-\mu)(x-\varphi(x)+\nabla g(x) y)+\mu\left(x-x^{(0)}\right)=0 \\
& \boldsymbol{Y g}(\boldsymbol{x})-\boldsymbol{\mu} \boldsymbol{Y}^{(0)} \boldsymbol{g}\left(\boldsymbol{x}^{(0)}\right)=0
\end{aligned}
$$

The limit set $T \subset \Omega \times R_{+}^{m} \times\{0\}$ is nonempty, and the $x$-component of any point in $T$ is a fixed point of $\boldsymbol{\varphi}(\boldsymbol{x})$ in $\Omega$, where $\boldsymbol{\mu} \in(0,1]$ is a homotopy parameter.

Algorithm for the Pathway Tracing Method. The theorem 1 shows that the combined homotopy interior-point method is a useful tool for solving the fixed-point problem which can be extended to non-convex set of definition of variables. A continuation homotopy pathway tracing method is used to find a global optimal solution based on the numerical method.

The predictor corrector numerical method is applied to trace the homotopy pathway $[5,6]$. The pathway is modeled by allowing both $\boldsymbol{x}$ and $\mu$ to be functions of an independent variable $s$ that represents arc length along the path. That is, $(\boldsymbol{x}(s), \boldsymbol{\mu}(s))$ is the point we arrive at by traveling a distance $s$ along the path from the initial point $(\boldsymbol{x}(0), \boldsymbol{\mu}(0))=(\boldsymbol{x}(0), 1)$ and $(s))$ is the tangent vector. Given a current point $(\boldsymbol{x}, \boldsymbol{\mu})$, we compute the tangent vector ( 1 , and take a small step (of length $\varepsilon$, say) along this direction to produce a predictor point $\left(\boldsymbol{x}^{P}, \boldsymbol{\mu}^{P}\right)$

$$
\left(\boldsymbol{x}^{P}, \boldsymbol{\mu}^{P}\right)=(\boldsymbol{x}, \boldsymbol{\mu})+\boldsymbol{\varepsilon}(\boldsymbol{\alpha}) .
$$


Usually, this new point will not lie exactly on the homotopy pathway, so some corrector iterations are applied to bring it back to the pathway, thereby identifying a new iterate $\left(\boldsymbol{x}^{+}, \boldsymbol{\mu}^{+}\right)$that satisfies the Eq.8 for the zero-path. When iterating, $\mu \rightarrow 0$, the limit point $x^{*}$ is the fixed point in $\Omega$.

\section{Numerical Example}

For a facility location problem between the urban inner and outer ring expressway, the definition set $\Omega=\left\{\boldsymbol{x} \in R^{2}:-x_{1}^{2}+10 x_{1}-x_{2}^{2}+10 x_{2}-46 \leq 0, x_{1}^{2}-10 x_{1}+x_{2}^{2}-10 x_{2}+25 \leq 0\right\}$ is non-convex. The coordinates of the customers is $C=\{(1,6),(2,2),(4,2),(3,8),(6,5),(7,6),(8,3),(6,10),(10,8)\}$. The corresponding demands is $W=\{15,5,18,7,12,15,13,10,15\}$. All the corresponding freight rate and is identical, $\boldsymbol{\rho}=1.0, r_{i}=1.0(i=1, \mathrm{~K}, n)$.

When the initial of $\boldsymbol{x}(1)$ is $(2,3)$, the predictor corrector numerical method is applied to trace the homotopy pathway. When iteration is the sixth, $\mu \rightarrow 0$, the limit point $\boldsymbol{x}^{*}=\boldsymbol{x}(0)=(3.097,4.386)$ is the fixed point in $\Omega$. The minimum objective $t(3.097,4.386)$ is 395.26 .

\section{Conclusions}

The facility location problem can be modeled by a fixed point problem. The hard point is that the collection of alternative sites is non-convex set while solving facility location problem between urban inner and outer ring expressway. The predictor corrector numerical method is applied to trace the regular solution curve of homotopy pathway and get the computational result. Numerical example shows that the numerical method by tracing the homotopy pathway with predictor-corrector is an effective method to solving the facility location problem and a global optimal solution can be obtained.

\section{Acknowledgements}

This research was financially supported by the Logistics \& Supply Chain Management Discipline Cultivating Program of Shanghai Second Polytechnic University (XXKPY1606).

\section{References}

[1] Z. Drezner, Facility location: a survey of applications and methods, Springer-Verlag, New York, 1995.

[2] D. L. Huff, Parameter estimation in the Huff model. Arc User, 11 (2003) 34-36.

[3] E. Cascetta, Transportation Systems Analysis, Springer Optimization and Its Applications 29, New York, 2009.

[4] E.L. Allgower and K. Georg, Numerical Continuation Methods: An Introduction, SpringerVerlag, Berlin, New York, 1990.

[5] J. Nocedal and S. J. Wright, Numerical Optimization, Springer-Verlag, New York 1999.

[6] B. Yu and Z. Lin, Homotopy method for a class of nonconvex Brouwer fixed point problems, Applied Mathematics and Computation, 74 (1996) 65-77.

[7] Q. Xu: Interior-point homotopy method for solving nonlinear programing and variational inequalities, doctoral dissertation, University of Jilin, Changchun 2003. 